\title{
COMMERCIAL PHOTODIODE SUITABILITY FOR SOLAR SIMULATOR LIGHT MONITORING
}

\author{
C. Barber ${ }^{(1)}$, P. López ${ }^{(2)}$, G. Martínez ${ }^{(1)}$ \\ (l) INTA-SPASOLAB, Carretera de Ajalvir km. 4, 28850 Torrejón de Ardoz, Spain \\ Email: barberdcl@inta.es, martinezfg@inta.es \\ (2) ISDEFE Consulting, C/ Beatriz de Bobadilla 3, 28040 Madrid, Spain,Email: lopezmp.pers_externo@inta.es
}

\begin{abstract}
The use of commercially available photodiodes for developing diverse solar simulator light monitoring applications is a very convenient approach due to availability, quality, performance and cost effectiveness.

Photodiodes as light monitors are meant to be operated in constant current zone, commonly referred to as photoconductive mode. In this operation mode, they offer linear response to impinging light over a few orders of magnitude. However, while presenting desirable characteristics in the manufacturer's specification sheets, there is a general lack of information regarding current response saturation for higher irradiances as it is the case of placing the photodiode directly under AM0 like conditions leading to possible misuse.
\end{abstract}

This paper will provide a guided approach on how to determine photodiode eligibility for solar simulator light monitoring applications. Needing to be linear around AM0 irradiances, suitability will rely on empirical IV tests at different irradiation conditions of three commercial silicon photodiodes performed in one of Spasolab's Xe based solar simulators. Further testing data including spectral response will also be provided from a specially manufactured off the production line photodiode from Hamamatsu, which has never been published, proving it to be an excellent option. Test data will serve to give guidelines on suitability for the application focusing on series resistance, having enough reverse bias headroom and containing power consumption to avoid excessive self-heating, yielding an appropriate bias voltage to assure linearity. Finally, a discussion on the photodiode's upper saturation limit determination will be introduced.

\section{INTRODUCTION}

Photodiodes are an interesting option in solar simulator behaviour characterization in applications such as short and long term stability metering and irradiance uniformity mapping. To do so, photodiodes have to be operated in constant current operation zone, or what is normally referred to photoconductive operation(see fig.
1), normally presenting near constant current due to high shunt resistances in Silicon.

Higher shunt resistance is better as in the case of solar cell performance, since it implies higher output current to bias voltage rejection. However, photodiodes are prone to saturation under AM0 irradiance conditions. This mainly has to do with the fact that photodiodes are manufactured to be high sensibility light sensors for a wide variety of applications and not optimized for carrier extraction under high irradiances (see fig. 1). This is a major difference with solar cells, which have much more complex and dense metallization grids, providing lower series resistances for the carrier paths.

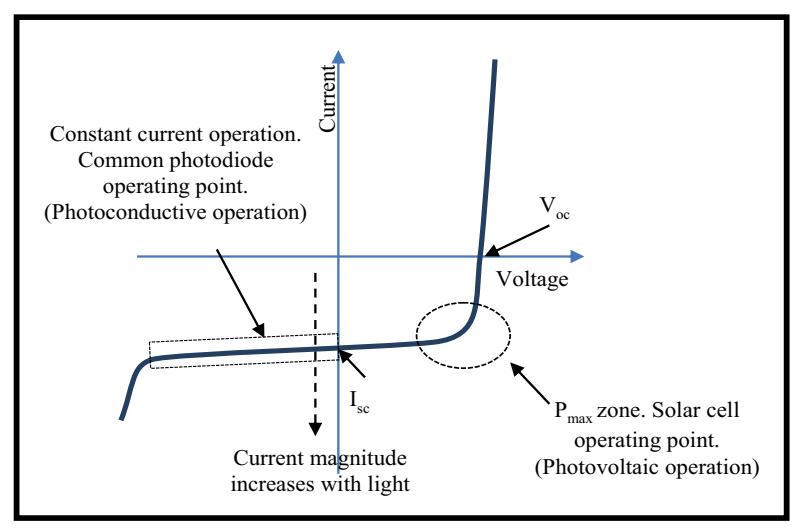

Figure 1 Comparing Solar Cell and Photodiode operating points on an IV characteristic

In practice, current saturation under a certain voltage operating point is originated by the lower density metallization grid found in the photodiodes which increases the series resistance found at the terminals of the device, as can be seen in fig. 2. Having the photodiode self-biased by driving its photocurrent through a low resistance is not enough in most applications. The photodiode's upper linear limit can be expanded by increasing reverse bias, however, doing so will make the photodiode consume power translating in self-heating of the device, which under higher irradiations and without active temperature control could quickly surpass temperature operational limits. 


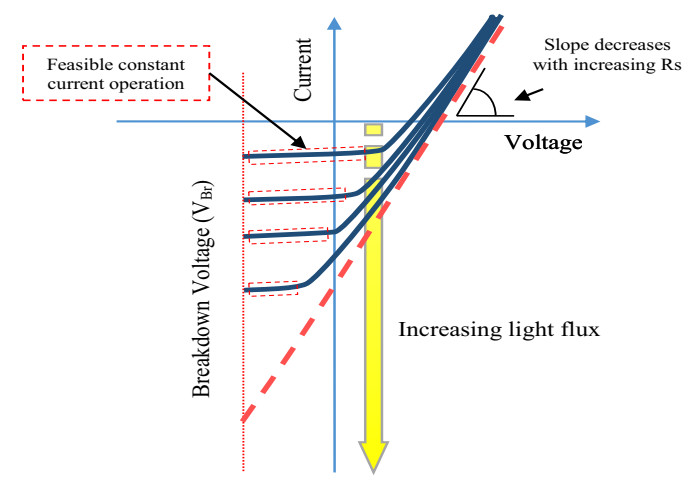

Figure2 Photoconductive zone reduction with increasing light flux

However, employing commercial photodiodes for such purposes presents good benefits for solar cell testing facilities highlighting their availability, cost, variety and overall robustness.

According to the previous, the work presented will explore the suitability of photodiodes for AM0 like irradiance conditions, determining appropriate working operation points and assuring linearity in a desired irradiance range. Furthermore, it is interesting to establish a way to determine an upper linear limit by extracting the photodiode's series resistance and shunt resistance from a measured IV curve under darkness. For the latter, parameter extraction and curve generation will be performed with IVFIT, comparing it to the measured IV curves. Although focusing on silicon photodiodes, which do have a broad spectral response range from 300 to $1100 \mathrm{~nm}$ (being sensible to the simulator zones that address top and various centre junctions), the approach is analogous for infrared InGaAs or Ge based photodiodes.

\section{DUT AND TEST SETUP DESCRIPTION}

\subsection{Device under test}

Three silicon photodiodes are compared in this work.

- Centronics OSD 100-6 (RS303-674 alternate coding). Circular $1 \mathrm{~cm}^{2}$ effective area, metal encapsulation with unspecified window material.

- Hamamatsu s1387-1010BQ. $1 \mathrm{~cm}^{2}$ effective area, enhanced UV response photodiode. Quartz window with ceramic encapsulation

- Hamamatsu s2387-1010R. Square $1 \mathrm{~cm}^{2}$ effective area. Resin cover with ceramic encapsulation.

- Modified off the production line Hamamatsu s2387-1010x. $1 \mathrm{~cm}^{2}$ effective area. Special request substituting epoxy resin by quartz cover with ceramic encapsulation.

\subsection{Test setup}

For this test, a triple source solar simulator at nominal conditions, employing only Xenon lamp and Spasolab's FEP [1] system will be employed. The data acquisition system (FEP) employs a calibrated Keithley 2602 sourcemeter to undertake electrical performance tests that are self triggered once light is shed upon the device, performing a fast IV sweep, setting IV sweep limits between $-3.5 \mathrm{~V}$ and the photodiode's Voc. This is the main characteristic of the FEP system and allows for lack of thermal control. The devices will suffer little temperature variation during the test and will initially be around laboratory conditions. The solar simulator will only be operated with the xenon source working at nominal power conditions. Irradiance steps will be made by using several neutral density filters (NDF).

For the spectral response data, SPASOLAB's double monochromator spectral response facility will be employed. Spectral response tests will only be performed to Hamamatsu's s2387-1010x.

\section{TEST RESULTS}

\subsection{Irradiance linearity data:}

Linearity tests have been separated in two due to photodiode availability at the time of the tests. The first group is comprised by S2387-1010R, S1337-1010BQ and Centronics OSD100-6 (with alternate code RS303674). Three mesh NDF filters of $3 \%, 11 \%$ and $43 \%$ have been introduced in the solar simulator which is set to near AM0 conditions, aiming to observe behaviour at operational injection levels. Second tests is performed over the tailored s2387-1010X, further increasing irradiance up to the maximum achievable without concentrating optics for the solar simulator employed, which sheds a total irradiance close to $1.5 \cdot \mathrm{AM} 0$. This is compared to Silicon primary reference standard. A better approach to determine linearity could have been performed by applying superposition of light sources [2]; unfortunately there was no option to do so. Despite not having adopted an orthodox linearity test approach, the main interest is to observe if substantial IV deformations appear at irradiance in the environment of AM0 to make the constant current zone disappear when swept in the defined voltage range.

Data for s1337-1010BQ is shown with an additional $10 \%$ transmission optical NDF due to the total loss of linear operation zone at the previous injections. The latter could have been tested up to higher reverse voltages to observe the appearance of constant current linear operation zone, however, this blue enriched silicon photodiode, due to its construction, has a rated reverse voltage breakdown of $-5 \mathrm{~V}$. 


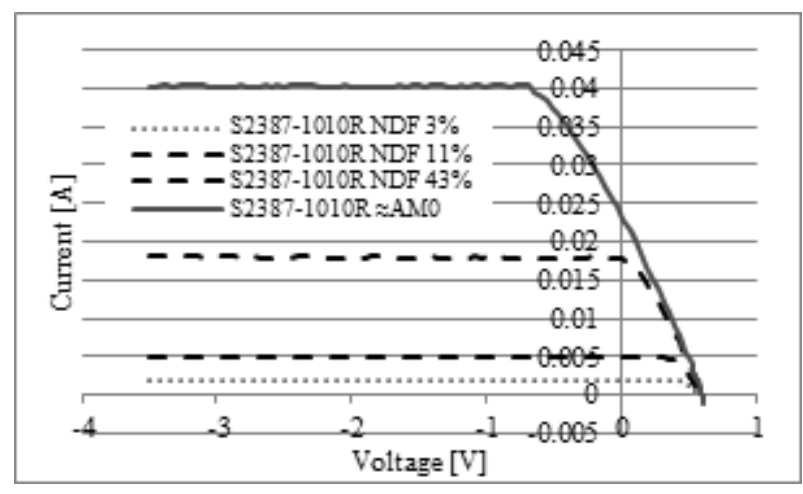

Figure3 S2387-1010R. Linearity Test IV Curves

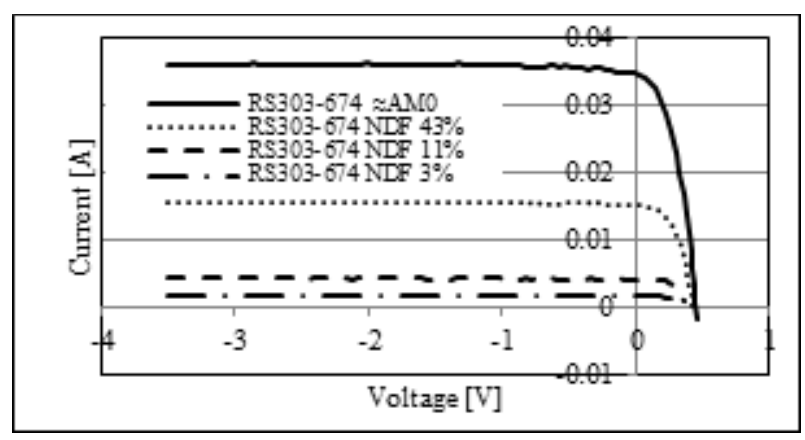

Figure4 RS303-674. Linearity Test IV Curves

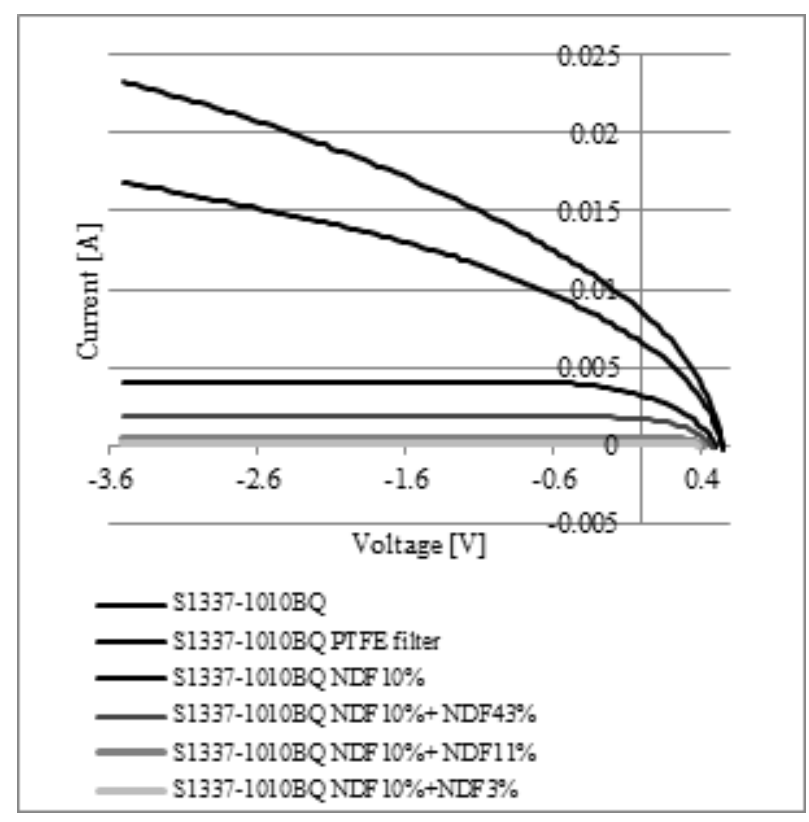

Figure5 s1337-1010BQ. Linearity Test IV curves

IV curve results in fig. 3 and fig. 4 show good form factor, with constant current zones entering the reverse bias region but with enough headroom to bias at low negative voltage levels.

For the blue enriched photodiode (see fig. 5), however, the IV curves tested prove it impossible to operate in linear operation zone as no constant current operation is seen and its breakdown voltage is near as aforementioned (this is due to the shallow junction required to trap higher energy photons).

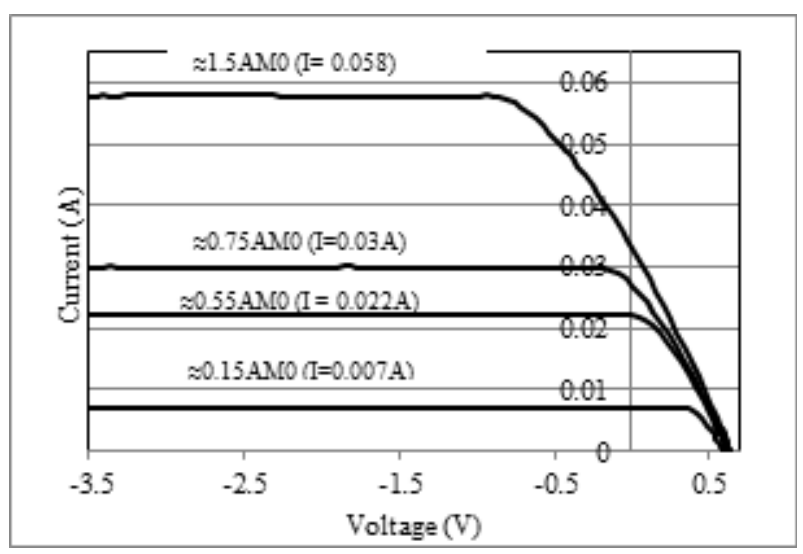

Figure6. s2387-1010X. Linearity Test IV curves

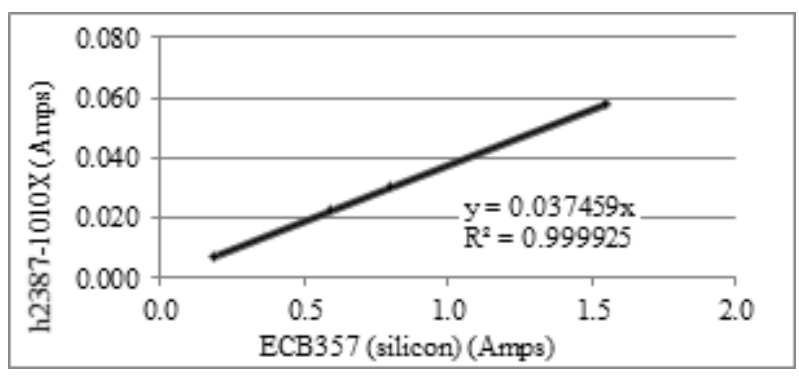

Figure 7 s2387-1010x vs Silicon Primary linearity data correlation

For the case of the specially fitted s2387 (see fig. 6), IV curves show constant current operation with enough headroom to increase irradiance up to $2 \mathrm{AM} 0$ if the photodiode is biased at $-3.5 \mathrm{~V}$, which is well under the breakdown voltage of the device. The linear correlation with the primary standard has an excellent match, noted by the R2 parameter as seen in fig. 7. Therefore, this photodiode makes a great irradiance monitoring device, despite the fact that it has to be operated in reverse voltage bias to do so.

\subsection{Spectral response:}

Spectral response for $2387-1010 \mathrm{x}$ has been tested between the range $280-1100 \mathrm{~nm}$. The starting point has been selected since it was feasible that the photodiode could provide output at such wavelengths having a quartz cover instead of the epoxy resin. For the standard commercial s2387-1010R, the spectral response is only provided down to $340 \mathrm{~nm}$. The measured spectral response is compared to the rest of the photodiodes, as seen in fig. 7, however the latter data is obtained from their respective datasheets by digitizing the provided curve. For better representation, all values have been interpolated from 190 to $1100 \mathrm{~nm}$ at $1 \mathrm{~nm}$ steps. 


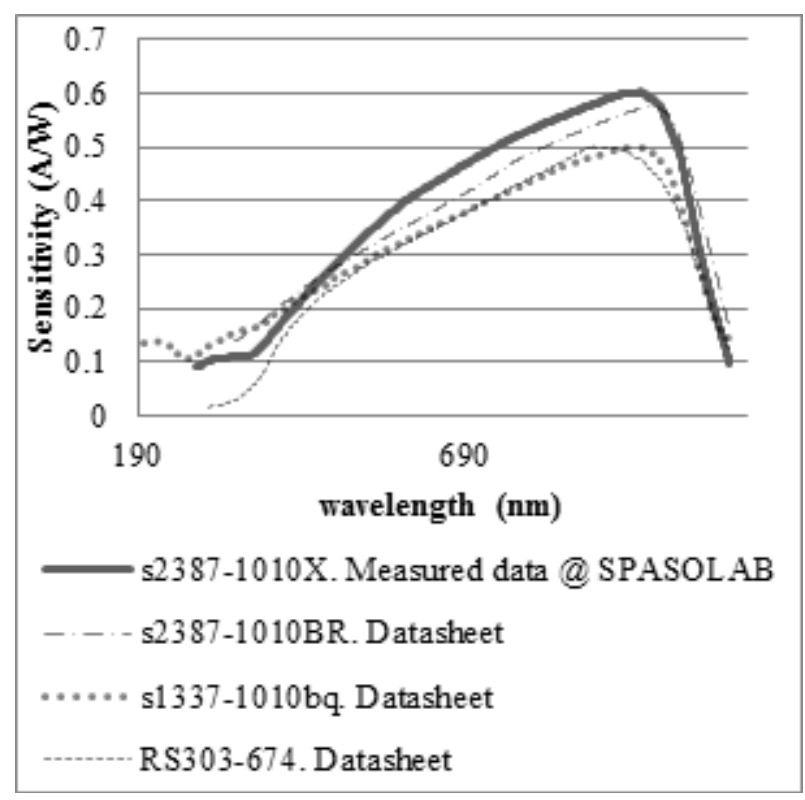

Figure 8. SR for 2387-1010X compared to the rest of photodiodes

Comparing the spectral response to that of s2387$1010 \mathrm{R}$, a general increase of spectral gain is obtained just by replacing epoxy resin by quartz. This however, does not affect the linear behaviour of the device, which has been proven linear even up to 1.5AM0 irradiances.

Having a quartz cover on silicon photodiodes for solar simulator monitoring applications is key to attain response under $300 \mathrm{~nm}$, as well as to avoid cover aging due to higher energy photons that affect epoxy resins. Further testing to increase the SR range could not be done, however, the interest of exploring the lower SR limit has not been overseen.

A total of four 2387-1010x photodiodes have been tested for SR in order to check consistency, the external quantum efficiency of all is shown in fig. 9. Curves show a close match with $40 \%$ EQE under $300 \mathrm{~nm}$.

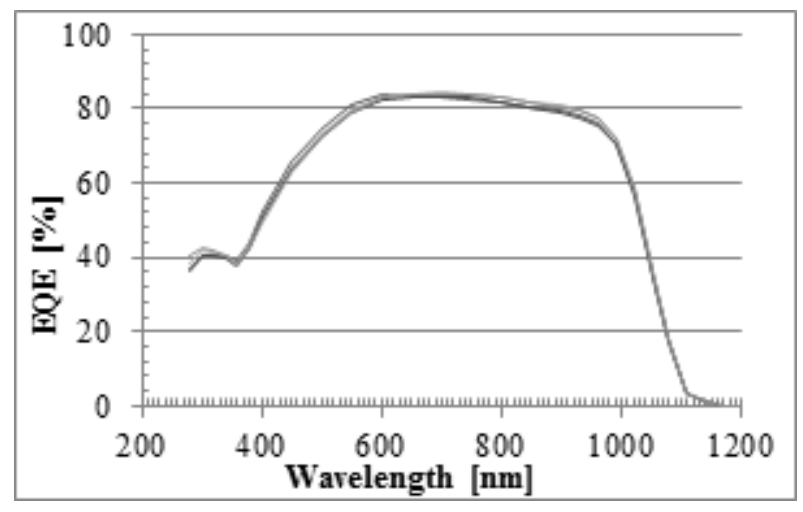

Figure 9. EQE of 4 different s2387-1010x photodiodes

\section{UPPER LINEARITY DISCUSSION}

When determining if a desired photodiode is suitable for an application that involves irradiances over $1000 \mathrm{~W} \cdot \mathrm{m}^{-}$ ${ }^{2}$, linear operation at such irradiance is the only concern.

From the manufacturer's literature and datasheets, it is not possible to extract an analytical conclusion, furthermore, they normally don't risk to include a figure that expresses suitability for high irradiances expressing high linearity but only up $10 \mathrm{~mW}$ over the photodiode area [3], as something applicable to silicon photodiodes. However, as seen from the IV data presented herein, this irradiance level can be heavily increased maintaining linear operation, even without requiring very high reverse voltage biasing.

The ultimate limiting parameter referred in the manufacturer's literature is series resistance. The effect of this parameter is quite straight forward, as it decreases the slope, shifting constant current operation to the $2^{\text {nd }}$ quadrant (when taking current flow as a generator).

A preliminary approach on this work regarded the extraction of the series resistance from a dark IV curve with IVFIT software [4], forecasting the operation with the corresponding current injection at AM0, employing a two diode lumped model. The latter was obtained by digitizing the datasheet SR graph and interpolating in nm steps (as mentioned for fig. 8) and AM0 spectrum by employing the current density expression Eq. ${ }^{\circ} 1$. The values obtained for the different photodiodes are gathered in tab. 1.

$$
\mathrm{J}_{@ \mathrm{AM} 0}=\int_{200}^{1100} \mathrm{SR}_{\mathrm{PD}}(\lambda) \cdot \mathrm{E}_{\mathrm{AM} 0}(\lambda) \cdot \mathrm{d} \lambda
$$

Table 1 Calculated current densities for each photodiode at AMO

\begin{tabular}{|l|r|}
\hline \multicolumn{1}{|c|}{ Photodiode } & I @ AM0 (A) \\
\hline s2387-1010X & $\mathbf{0 . 0 3 7 9 7}$ \\
\hline S2387-1010BR & $\mathbf{0 . 0 3 5 8 1}$ \\
\hline s1337-1010BQ & $\mathbf{0 . 0 3 2 8 4}$ \\
\hline rs303-674 & $\mathbf{0 . 0 3 0 8 5}$ \\
\hline
\end{tabular}

However, when generating a curve with the series resistance extracted from the dark IV curve, the modelled behaviour fails completely to recreate the tested data. By comparing the tested data series resistance in dark condition with that near AM0, the latter has an increased value of 1 decade, behaving in the same fashion for all photodiodes.

As series resistance has been observed to vary with the illumination level, there is no way to employ the general model to predict suitability with only employing a dark IV curve and parameters from the datasheet.

\subsection{Series Resistance variation with higher}




\section{current injection.}

The variation of the series resistance with the irradiance is an effect that has been described multiple times within the literature, and was first well described in 1963 [5]. This phenomenon was observed specifically when comparing IV curves of solar cells with low and high density metallization grid at increasing irradiances. In their work, the tests showed a heavy increase in the series resistance with illumination for the low density cell and not such a difference in the case of higher density, appearing rounding effects in the IV curves. This effect can clearly be seen in fig. 5, where the shape of the IV curve of s1337-1010BQ, ends up changing drastically.

Recent works have progressed in obtaining numerically such varying series resistances as found in [6]. However, it requires the previous acquisition of various IV curves up to high injection levels.

\subsection{Manufacturer's saturation limit notes}

The most commonly expressed definition for saturation in the manufacturer's literature is shown in Eq. ${ }^{\circ} 2$, which is taken from [3]. This is affected by the uncontrolled parameter Rs. This again, requires to be obtained prior to determining saturation, as aforementioned.

Also, Psat is suggested to be under $10 \mathrm{~mW}$ impinging the effective area of the device, which limits the use to $10 \%$ AM0 equivalent total irradiance. However, they also recommend increasing linearity by applying reverse voltages. This is a general definition; however, taking the s2387-1010 for example, it has been proven to operate in linear operation up to irradiances 15 times the limit.

$$
\begin{aligned}
& \text { Psat }=\frac{V_{B I}+V_{R}}{\left(R_{S}+R_{L}\right) \cdot S_{\lambda}} \cdots \\
& \text { Psat } \leq 10 \mathrm{~mW} \\
& \mathrm{~V}_{\mathrm{BI}-->} \text { Built in voltage } \\
& \mathrm{V}_{\mathrm{R}}=\text { Applied Reverse Voltage } \\
& \mathrm{S}_{\lambda-->\text { Sensitivity at wavelength } \lambda} \lambda
\end{aligned}
$$

Therefore, series resistance is a key parameter that affects the saturation limit of the device, however it still requires to be obtained previously.

\section{PHOTODIODE ELIGIBILITY}

Taking into account the reverse breakdown voltage and maximum operation temperature ratings supplied by the manufacturer, linear operation will be defined as having enough reverse voltage breakdown overhead to bias the photodiode in constant current zone at the defined irradiance. To keep within safe operation conditions, bias voltage will have to be within $80 \%$ of the reverse bias voltage and special considerations will be made taking into consideration the dissipated power within the junction. However, upper irradiance level has not been possible to determine from the information provided in the datasheets, since series resistance needs to be measured before. This, however, can be performed without sophisticated light sources, just enough to provide high current injection levels. The current injection level at AM0 can be determined with the digitized SR response from the datasheet and the standard AM0, and light, from a simple source, can be increased until those current levels are met. This inconvenience wouldn't exist if manufacturers included series resistance as a function of light intensity or their maximum light intensity operation points at different reverse bias levels.

\section{CONCLUSIONS AND FUTURE WORKS}

In this paper we place focus on determining commercial photodiode suitability for Multiple Zone solar simulator monitoring by introducing a series of irradiance linearity verification and operation point guidelines. Linearity tests by performing IV curves at different irradiances have been performed on four silicon photodiodes, comparing linearity to that of a silicon reference standard. Intending to forecast irradiance linearity upper limit without the need of performing previous tests has been deemed not possible with the current information provided by the manufacturers, since the series resistance varies heavily with the irradiance on devices that have low metallization density. This effect has been contrasted with the literature, finding that previous IV curve knowledge is required. However, only current injection is required to obtain the desired series resistance, therefore, linearity can be tested with simple halogen lamps that provide enough power to recreate desired operation points.

Additionally, results for a tailored photodiode have been presented and its suitability for solar simulator monitoring when biased actively under $-1 \mathrm{~V}$ has been shown.

\section{ACKOWLEDGEMENTS}

The research was co-supported by the European Space Agency (ESA) under contract No. 4000107040/12/NL/GLC and the European Metrology Research Programme under the project ENG 55 (Photoclass). The EMRP is jointly funded by the EMRP participating countries within EURAMET and the European Union. 


\section{REFERENCES}

1. C. Barber and A. Gras (2014), "SPASOLAB's Low Temperature Solar Array Coupon Electrical Performance under Continuous Light Test Setup," in 10th European Space Power Conference (ESPC), Noordwijkerhout, Netherlands.

2. K. Emery, S. Winter, S. Pinegar and D. Nalley (2006), "Linearity Testing of Photovoltaic Cells," in 4th World Conference on Photovoltaic Energy Conversion (WCPEC-4) IEEE, Waikoloa.

3. Hamamatsu Corporation (2014), OptoSemiconductor Handbook. Chapter $2 \mathrm{Si}$ Photodiode, Hamamatsu Photonics Solid State Division.

4. Energy Research Centre of the Netherlands (ECN), "IVFIT curve fitting program," ECN, 2015. [Online]. Available: https://www.ecn.nl/units/zon/productsservices/i-v-curve-fitting-program-ivfit. [Accessed february 2016].

5. H. R. M. Wolf (1961), "Series resistance effects on solar cell measurements," in Pacific General Meeting of the AIEE, Salt Lake City, Utah.

6. T. Luka, S. Eiternick and M. Turek (2014), "Determination of the Current and illuminatino dependent series resistance using LED Solar Simulators," in 29th European Photovoltaic Solar Energy Conference and Exhibition, Amsterdam, NL. 